\title{
ERRATUM
}

\section{Reverse Genetics System for Porcine Enteric Calicivirus, a Prototype Sapovirus in the Caliciviridae}

\author{
Kyeong-Ok Chang, ${ }^{1 *}$ Stanislav V. Sosnovtsev, ${ }^{1}$ Gaël Belliot, ${ }^{1}$ Qiuhong Wang, ${ }^{2}$ \\ Linda J. Saif, ${ }^{2}$ and Kim Y. Green ${ }^{1}$
}

Laboratory of Infectious Diseases, National Institute of Allergy and Infectious Diseases, National Institutes of Health, Bethesda, Maryland, ${ }^{1}$ and Food Animal Health Research Program, Department of Veterinary Preventive Medicine, The Ohio State University, Wooster, Ohio ${ }^{2}$

Volume 79, no. 3, p. 1409-1416, 2005. Page 1409: The byline should appear as shown above. 